\title{
A comparative study of some methods for color medical images segmentation
}

\author{
Liana Stanescu*, Dumitru Dan Burdescu and Marius Brezovan
}

\begin{abstract}
The aim of this article is to study the problem of color medical images segmentation. The images represent pathologies of the digestive tract such as ulcer, polyps, esophagites, colitis, or ulcerous tumors, gathered with the help of an endoscope. This article presents the results of an objective and quantitative study of three segmentation algorithms. Two of them are well known: the color set back-projection algorithm and the local variation algorithm. The third method chosen is our original visual feature-based algorithm. It uses a graph constructed on a hexagonal structure containing half of the image pixels in order to determine a forest of maximum spanning trees for connected component representing visual objects. This third method is a superior one taking into consideration the obtained results and temporal complexity. These three methods were successfully used in generic color images segmentation. In order to evaluate these segmentation algorithms, we used error measuring methods that quantify the consistency between them. These measures allow a principled comparison between segmentation results on different images, with differing numbers of regions generated by different algorithms with different parameters.
\end{abstract}

Keywords: graph-based segmentation, color segmentation, segmentation evaluation, error measures

\section{Introduction}

The problem of partitioning images into homogenous regions or semantic entities is a basic problem for identifying relevant objects. Some of the practical applications of image segmentation are medical imaging, locate objects in satellite images (roads, forests, etc.), face recognition, fingerprint recognition, traffic control systems, visual information retrieval, or machine vision.

Segmentation of medical images is the task of partitioning the data into contiguous regions representing individual anatomical objects. This task is vital in many biomedical imaging applications such as the quantification of tissue volumes, diagnosis, localization of pathology, study of anatomical structure, treatment planning, partial volume correction of functional imaging data, and computer-integrated surgery $[1,2]$.

This article presents the results of an objective and quantitative study of three segmentation algorithms.

Two of them are already well known:

\footnotetext{
* Correspondence: stanescu@software.ucv.ro

Faculty of Automation, Computers and Electronics, University of Craiova, 200440, Romania
}

\section{Springer

(c) 2011 Stanescu et al; licensee Springer. This is an Open Access article distributed under the terms of the Creative Commons Attribution License (http://creativecommons.org/licenses/by/2.0), which permits unrestricted use, distribution, and reproduction in any medium, provided the original work is properly cited.
- The color set back-projection; this method was implemented and tested on a wide variety of images including medical images and has achieved good results in automated detection of color regions (CS).

- An efficient graph-based image segmentation algorithm known also as the local variation algorithm (LV)

The third method design by us is an original visual feature-based algorithm that uses a graph constructed on a hexagonal structure (HS) containing half of the image pixels in order to determine a forest of maximum spanning trees for connected component representing visual objects. Thus, the image segmentation is treated as a graph partitioning problem.

The novelty of our contribution concerns the HS used in the unified framework for image segmentation and the using of maximum spanning trees for determining the set of nodes representing the connected components.

According to medical specialists most of digestive tract diseases imply major changes in color and less in texture of the affected tissues. This is the reason why we have chosen to do a research of some algorithms that realize images segmentation based on color feature. 
Experiments were made on color medical images representing pathologies of the digestive tract. The purpose of this article is to find the best method for the segmentation of these images.

The accuracy of an algorithm in creating segmentation is the degree to which the segmentation corresponds to the true segmentation, and so the assessment of accuracy of segmentation requires a reference standard, representing the true segmentation, against which it may be compared. An ideal reference standard for image segmentation would be known to high accuracy and would reflect the characteristics of segmentation problems encountered in practice [3].

Thus, the segmentation algorithms were evaluated through objective comparison of their segmentation results with manual segmentations. A medical expert made the manual segmentation and identified objects in the image due to his knowledge about typical shape and image data characteristics. This manual segmentation can be considerate as "ground truth".

The evaluation of these three segmentation algorithms is based on two metrics defined by Martin et al.: Global Consistency Error (GCE), and Local Consistency Error (LCE) [4]. These measures operate by computing the degree of overlap between clusters or the cluster associated with each pixel in one segmentation and its "closest" approximation in the other segmentation. GCE and LCE metrics allow labeling refinement in either one or both directions, respectively.

The comparative study of these methods for color medical images segmentation is motivated by the following aspects:

- The methods were successfully used in generic color images segmentation

- The CS algorithm was implemented and studied for color medical images segmentation, the results being promising [5-8]

- There are relatively few published studies for medical color images of the digestive tract, although the number of these images, acquired in the diagnostic process, is high

- The color medical images segmentation is an important task in order to improve the diagnosis and treatment activity

- There is not a segmentation method for medical images that produces good results for all types of medical images or applications.

The article is organized as follows: Section 2 presents the related study; Section 3 describes our original method based on a HS. Sections 4 and 5 briefly present the other two methods: the color set back-projection and the LV; Section 6 describes the two error metrics used for evaluation; Section 7 presents the experimental results and Section 8 presents the conclusion of this study.

\section{Related study}

Image segmentation is defined as the partitioning of an image into no overlapping, constituent regions that are homogeneous, taking into consideration some characteristic such as intensity or texture [1,2].

If the domain of the image is given by $I$, then the segmentation problem is to determine the sets $S_{k} \subset I$ whose union is the entire image. Thus, the sets that make up segmentation must satisfy:

$$
I=\bigcup_{k=1}^{K} S_{k}
$$

Where $S_{k} \cap S_{j}=\varnothing$ for $k \neq j$ and each $S_{k}$ is connected [9].

In an ideal mode, a segmentation method finds those sets that correspond to distinct anatomical structures or regions of interest in the image.

Segmentation of medical images is the task of partitioning the data into contiguous regions representing individual anatomical objects. This task plays a vital role in many biomedical imaging applications: the quantification of tissue volumes, diagnosis, localization of pathology, study of anatomical structure, treatment planning, partial volume correction of functional imaging data, and computer-integrated surgery.

Segmentation is a difficult task because in most cases it is very hard to separate the object from the image background. Also, the image acquisition process brings noise in the medical data. Moreover, inhomogeneities in the data might lead to undesired boundaries. The medical experts can overcome these problems and identify objects in the data due to their knowledge about typical shape and image data characteristics. But, manual segmentation is a very time-consuming process for the already increasing amount of medical images. As a result, reliable automatic methods for image segmentation are necessary.

It cannot be said that there is a segmentation method for medical images that produces good results for all types of images. There have been studied several segmentation methods that are influenced by factors such as application domain, imaging modality, or others $[1,2,10]$.

The segmentation methods were grouped into categories. Some of these categories are thresholding, region growing, classifiers, clustering, Markov random field (MRF) models, artificial neural networks (ANNs), deformable models, or graph partitioning. Of course, there are other important methods that do not belong to any of these categories [1]. 
In thresholding approaches, an intensity value called the threshold must be established. This value will separate the image intensities in two classes: all pixels with intensity greater than the threshold are grouped into one class and all the other pixels into another class. If more than one threshold is determined, the process is called multi-thresholding.

Region growing is a technique for extracting a region from an image that contains pixels connected by some predefined criteria, based on intensity information and/ or edges in the image. In its simplest form, region growing requires a seed point that is manually selected by an operator, and extracts all pixels connected to the initial seed having the same intensity value. It can be used particularly for emphasizing small and simple structures such as tumors and lesions $[1,11]$.

Classifier methods represent pattern recognition techniques that try to partition a feature space extracted from the image using data with known labels.

A feature space is the range space of any function of the image, with the most common feature space being the image intensities themselves. Classifiers are known as supervised methods because they need training data that are manually segmented by medical experts and then used as references for automatically segmenting new data $[1,2]$.

Clustering algorithms work like classifier methods but they do not use training data. As a result they are called unsupervised methods. Because there is not any training data, clustering methods iterate between segmenting the image and characterizing the properties of each class. It can be said that clustering methods train themselves using the available data $[1,2,12,13]$.

MRF is a statistical model that can be used within segmentation methods. For example, MRFs are often incorporated into clustering segmentation algorithms such as the $K$-means algorithm under a Bayesian prior model. MRFs model spatial interactions between neighboring or nearby pixels. In medical imaging, they are typically used to take into account the fact that most pixels belong to the same class as their neighboring pixels. In physical terms, this implies that any anatomical structure that consists of only one pixel has a very low probability of occurring under a MRF assumption [1,2].

ANNs are massively parallel networks of processing elements or nodes that simulate biological learning. Each node in an ANN is capable of performing elementary computations. Learning is possible through the adaptation of weights assigned to the connections between nodes [1,2]. ANNs are used in many ways for image segmentation.

Deformable models are physically motivated, modelbased techniques for outlining region boundaries using closed parametric curves or surfaces that deform under the influence of internal and external forces. To outline an object boundary in an image, a closed curve or surface must be placed first near the desired boundary that comes into an iterative relaxation process [14-16].

To have an effective segmentation of images using varied image databases the segmentation process has to be done based on the color and texture properties of the image regions $[10,17]$.

The automatic segmentation techniques were applied on various imaging modalities: brain imaging, liver images, chest radiography, computed tomography, digital mammography, or ultrasound imaging $[1,18,19]$.

Finally, we briefly discuss the graph-based segmentation methods because they are most relevant to our comparative study.

Most graph-based segmentation methods attempt to search a certain structures in the associated edge weighted graph constructed on the image pixels, such as minimum spanning tree [20,21], or minimum cut $[22,23]$. The major concept used in graph-based clustering algorithms is the concept of homogeneity of regions.

For color segmentation algorithms, the homogeneity of regions is color-based, and thus the edge weights are based on color distance. Early graph-based methods [24] use fixed thresholds and local measures in finding a segmentation.

The segmentation criterion is to break the minimum spanning tree edges with the largest weight, which reflect the low-cost connection between two elements. To overcome the problem of fixed threshold, Urquhar [25] determined the normalized weight of an edge using the smallest weight incident on the vertices touching that edge. Other methods $[20,21]$ use an adaptive criterion that depends on local properties rather than global ones. In contrast with the simple graph-based methods, cut-criterion methods capture the non-local properties of the image. The methods based on minimum cuts in a graph are designed to minimize the similarity between pixels that are being split $[22,23,26]$. The normalized cut criterion [22] takes into consideration self-similarity of regions. An alternative to the graph cut approach is to look for cycles in a graph embedded in the image plane. For example in [27], the quality of each cycle is normalized in a way that is closely related to the normalized cuts approach.

Other approaches to image segmentation consist of splitting and merging regions according to how well each region fulfills some uniformity criterion. Such methods [28,29] use a measure of uniformity of a region.

In contrast, [20,21] use a pairwise region comparison rather than applying a uniformity criterion to each individual region. A number of approaches to segmentation are based on finding compact clusters in some feature 
space [30,31]. A recent technique using feature space clustering [30] first transforms the data by smoothing it in a way that preserves boundaries between regions.

Our method is related to the works in $[20,21]$ in the sense of pairwise comparison of region similarity. We use different measures for internal contrast of a connected component and for external contrast between two connected components than the measures used in $[20,21]$. The internal contrast of a component $C$ represents the maximum weight of edges connecting vertices from $\mathrm{C}$, and the external contrast between two components represents the maximum weight of edges connecting vertices from these two components. These measures are in our opinion closer to the human perception. We use maximum spanning tree instead of minimum spanning tree in order to manage external contrast between connected components.

\section{Image segmentation using an HS}

The low-level system for image segmentation described in this section is designed to be integrated in a general framework of indexing and semantic image processing. In this stage, it uses color to determine salient visual objects.

The color is the visual feature that is immediately perceived on an image. There is no color system that is universally used, because the notion of color can be modeled and interpreted in different ways. Each system has its own color models that represent the system parameters.

There exist several color systems, for different purposes: RGB (for displaying process), XYZ (for color standardization), rgb, xyz (for color normalization and representation), CieL"u*v*, CieL"a*b* (for perceptual uniformity), HSV (intuitive description) [2,32].

We decided to use the RGB color space because it is efficient and no conversion is required. Although it also suffers from the non-uniformity problem where the same distance between two color points within the color space may be perceptually quite different in different parts of the space, within a certain color threshold it is still definable in terms of color consistency. We use the perceptual Euclidean distance with weight-coefficients (PED) as the distance between two colors, as proposed in [33]:

$$
\operatorname{PED}(e, u)=\sqrt{w_{R}\left(R_{e}-R_{u}\right)^{2}+w_{G}\left(G_{e}-G_{u}\right)^{2}+w_{B}\left(B_{e}-B_{u}\right)^{2}}
$$

the weights for the different color channels, $w_{R}, w_{G}$, and $w_{B}$ verify the condition $w_{R}+w_{G}+w_{B}=1$.

Based on the theoretical and experimental results on spectral and realworld datasets, in [25] it is concluded that the PED distance with weightcoefficients $\left(w_{R}=\right.$ $\left.0.26, w_{G}=0.70, w_{B}=0.04\right)$ correlates significantly higher than all other distance measures including the angular error and Euclidean distance.

In order to optimize the running time of segmentation and contour detection algorithms, we use a HS constructed on the image pixels, as presented in Figure 1.

Each hexagon represents an elementary item and the entire HS represents a grid-graph, $G=(V, E)$, where each hexagon $h$ in this structure has a corresponding vertex $v \in V$. The set $E$ of edges is constructed by connecting pairs of hexagons that are neighbors in a 6-connected sense, because each hexagon has six neighbors.

The advantage of using hexagons instead of pixels as elementary piece of information is that the amount of memory space associated to the graph vertices is reduced. Denoting by $n_{p}$ the number of pixels of the initial image, the number of the resulted hexagons is always less than $n_{p}=4$, and then the cardinal of both sets $V$ and $E$ is significantly reduced.

We associate to each hexagon $h$ from $V$ two important attributes representing its dominant color and the coordinates of its gravity center. For determining these attributes, we use eight pixels contained in a hexagon h: six pixels from the frontier and two interior pixels. We select one of the two interior pixels to represent with approximation the gravity center of the hexagon because pixels from an image have integer values as coordinates. We select always the left pixel from the two interior pixels of a hexagon $h$ to represent the pseudo-center of the gravity of $h$, denoted by $g(h)$.

The dominant color of a hexagon is denoted by $c(h)$ and it represents the mean color vector of the all eight colors of its associated pixels. Each hexagon $h$ in the hexagonal grid is thus represented by a single point, $g$ (h),6 having the color $c(h)$.

The segmentation system creates an HS on the pixels of the input image and an undirected grid graph having hexagons as vertices, and uses this graph in order to produce a set of salient objects contained in the image. In order to allow an unitary processing for the multilevel system at this level we store, for each determined component $C$ :

- an unique index of the component;

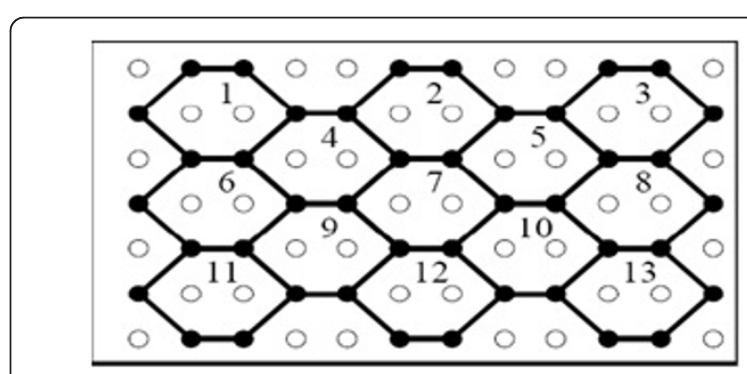

Figure 1 HS constructed on the image pixels. 
- the set of the hexagons contained in the region associated to $C$;

- the set of hexagons located at the boundary of the component.

In addition for each component a mean color of the region is extracted. Our HS is similar to quincunx sampling scheme, but there are some important differences. The quincux sample grid is a sublattice of a square lattice that retains half of the image pixels [34]. The key point of our HS, that also uses half of the image pixels, is that the hexagonal grid is not a lattice because hexagons are not regular. Although our hexagonal grid is not a hexagonal lattice, we use some of the advantages of the hexagonal grid such as uniform connectivity. In our case, only one type of neighborhood is possible, sixth neighborhood structure, unlike several types as N4 and N8 in the case of square lattice.

\subsection{Algorithms for computing the color of a hexagon and the list of hexagons with the same color}

The algorithms return the list of salient regions from the input image. This list is obtained using the hexagonal network and the distance between two colors in the RGB color space. In order to obtain the color of a hexagon a procedure called sameVertexColour is used. This procedure has a constant execution time because all calls are constant in time processing. The color information will be used by the procedure expandColorArea to find the list of hexagons that have the same color.

\subsubsection{Determination of the hexagon color}

The input of this procedure contains the current hexagon $h_{i}, L_{1}$-the colors list of pixels corresponding to the hexagonal network: $L_{1}=\left\{p_{1}, \ldots, p_{6 n}\right\}$. The output is represented by the object crtColorHexagon.

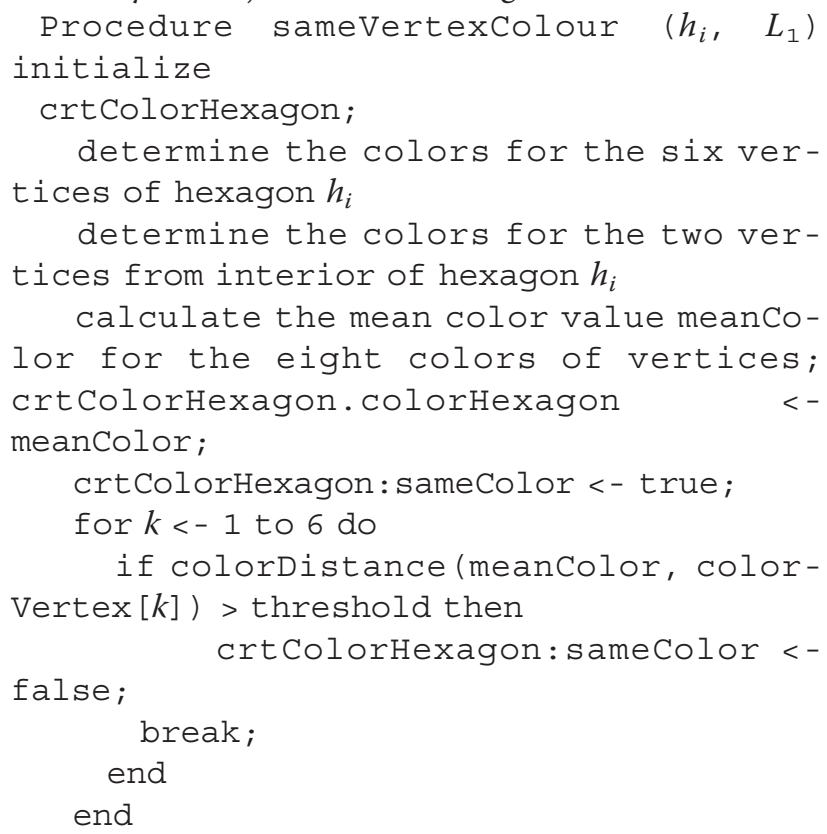

return crtColorHexagon;

In the above function, the threshold value is an adaptive one, defined as the sum between the average of the color distances associated to edges (between the adjacent hexagons) and the standard deviation of these color distances.

\subsubsection{Expand the current region}

The function expandColourArea is a depth-first traversing procedure, which starts with an specified hexagon $h_{i}$, pivot of a region item, and determines the list of all adjacent hexagons representing the current region containing $h_{i}$ such that the color dissimilarity between the adjacent hexagons is below a determined threshold.

The input parameters of this function is the current region item, index-CrtRegion, its first hexagon, $h_{i}$, and the list of all hexagons $V$ from the hexagonal grid.

Procedure expandColourArea $\left(h_{i}\right.$, crtRegionItem, $V)$

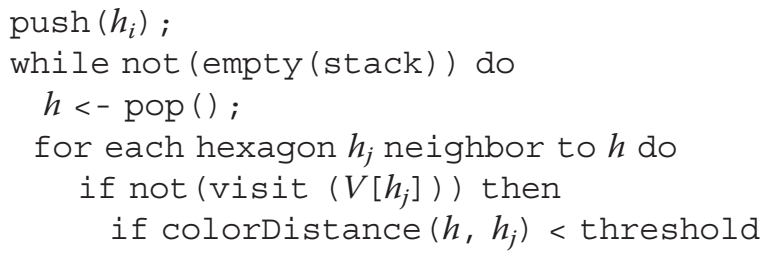

then

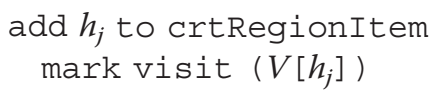

end

The running time of the procedure expandColourArea is $O(n)$ where $n$ is the number of hexagons from a region with the same color [35].

\subsection{The algorithm used to obtain the regions}

The procedures presented above are used by the listRegions procedure to obtain the list of regions.

This procedure has an input which contains the vector

$V$ representing the list of hexagons and the list $L_{1}$.

The output is represented by a list of colors pixels and a list of regions for each color.

Procedure listRegions $\left(V, L_{1}\right)$

ColourNb $<-0$;

for $i<-1$ to $n$ do

initialize crtRegionItem;

if not (visit $\left(h_{-} i\right)$ ) then

crtColorHexagon <- sameVertexCo-

$\operatorname{lour}\left(L_{1}, h_{i}\right)$;

if crtColorHexagon. sameColor then

$k<-$ findColor (crtColorHexagon.

color);

if $k<0$ then 
add new color ccolourNb to list $C$;

$k<-$ colourNb++ ;

indexCrtRegion $<-0$;

else

indexCrtColor $<-k$;

indexCrtRegion<-

findLast IndexRegion (index

Crtcolor):

indexCrtRegion++;

end

hi.indexRegion <- indexCrtRegion;

hi. indexColor <- $k$;

add $h_{i}$ to crtRegionItem;

expandColourArea $\left(h_{i}, L_{1}, \mathrm{~V}\right.$,

indexCrtRegion, indexCrtColor,

crtRegionItem);

add new region crtRegionItem to

list of element $k$ from $C$

end

end

end

The running time of the procedure list Regions is $O(n)$

${ }^{2}$, where $n$ is the number of the hexagons network [35].

Let $G=(V, E)$ be the initial graph constructed on the HS of an image. The color-based sequence of segmentations, $S_{i}=\left(S^{0}, S^{1}, \ldots, S^{t}\right)$, will be generated by using a color-based region model and a maximum spanning tree construction method based on a modified form of the Kruskal's algorithm [36].

In the color-based region model, the evidence for a boundary between two regions is based on the difference between the internal contrast of the regions and the external contrast between them. Both notions of internal contrast or internal variation of a component, and external contrast or external variation between two components are based on the dissimilarity between two colors [37]:

$$
\begin{aligned}
& \operatorname{ExtVar}\left(C^{\prime}, C^{\prime \prime}\right)=\max _{\left(h_{i}, h_{j}\right) \in c b\left(c^{\prime}, c^{\prime \prime}\right)} w\left(h_{i}, h_{j}\right) \\
& \operatorname{Int\operatorname {Var}}(C)=\max _{\left(h_{i}, h_{j}\right) \in c} w\left(h_{i}, h_{j}\right)
\end{aligned}
$$

where $c b\left(C^{\prime}, C^{\prime \prime}\right)$ represents the common boundary between the components $C^{\prime}$ and $C^{\prime \prime}$ and $w$ is the color dissimilarity between two adjacent hexagons:

$$
w\left(h_{i}, h_{j}\right)=\operatorname{PED}\left(c\left(h_{i}\right), c\left(h_{j}\right)\right)
$$

where $c(h)$ represents the mean color vector associated with the hexagon $h$.

The maximum internal contrast between two components is defined as follows [37]:

$$
\operatorname{IntVar}\left(C^{\prime}, C^{\prime \prime}\right)=\max \left(\operatorname{Int} \operatorname{Var}\left(C^{\prime}\right), \operatorname{Int} \operatorname{Var}\left(C^{\prime \prime}\right)\right)+r
$$

where the threshold $r$ is an adaptive value defined as the sum between the average of the color distances associated to edges and the standard deviation, $r=\mu+$ $\sigma$.

The comparison predicate between two neighboring components $C^{\prime}$ and $C^{\text {" }}$ determines if there is an evidence for a boundary between them [37].

$$
\text { dif } f_{\text {col }}\left(C^{\prime}, C^{\prime \prime}\right)=\left\{\begin{array}{l}
\text { true, ExtVar }\left(C^{\prime}, C^{\prime \prime}\right)>\operatorname{IntVar}\left(C^{\prime}, C^{\prime \prime}\right) \\
\text { false, ExtVar }\left(C^{\prime}, C^{\prime \prime}\right) \leq \operatorname{IntVar}\left(C^{\prime}, C^{\prime \prime}\right)
\end{array}\right.
$$

The color-based segmentation algorithm represents an adapted form of a Kruskal's algorithm and it builds a maximal spanning tree for each salient region of the input image.

\section{The color set back-projection algorithm}

Color sets provide an alternative to color histograms for representing color information. Their utilization is based on the assumption that salient regions have not more than few equally prominent colors [38].

The color set back-projection algorithm proposed in [38] is a technique for the automated extraction of regions and representation of their color content.

The back-projection process requires several stages: color set selection, back-projection onto the image, thresholding, and labeling. Candidate color sets are selected first with one color, then with two colors, etc., until the salient regions are extracted. For each image quantization of the RGB color space at 64 colors is performed.

The algorithm follows the reduction of insignificant color information and makes evident the significant CS, followed by the generation, in automatic way, of the regions of a single color, of the two colors, etc.

For each detected region the color set, the area and the localization are stored. The region localization is given by the minimal bounding rectangle. The region area is represented by the number of color pixels, and can be smaller than the minimum bounding rectangle.

The image processing algorithm computes both the global histogram of the image, and the binary color set $[7,32]$. The quantized colors are stored in a matrix. To this matrix a $5 \times 5$ median filter is applied which has the role of eliminating the isolated points. The process of regions extraction is using the filtered matrix and it is a depth-first traversal described in pseudo-code in the following way:

Procedure FindRegions (Image I, colorset C)
Initstack $(S)$
Visited $=\varnothing$ 


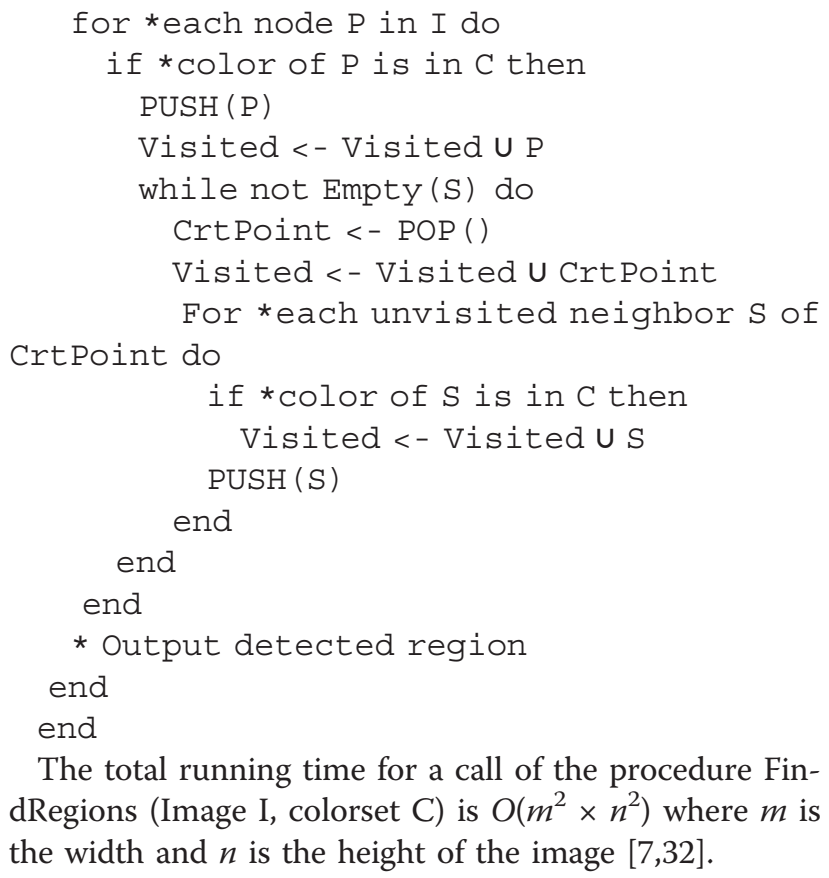

\section{Local variation algorithm}

This algorithm described in [20] is using a graph-based approach for the image segmentation process. The pixels are considered the graph nodes so in this way it is possible to define an undirected graph $G=(V, E)$ where the vertices $v_{i}$ from $V$ represent the set of elements to be segmented. Each edge $\left(v_{i}, v_{j}\right)$ belonging to $E$ has associated a corresponding weight $w\left(v_{i}, v_{j}\right)$ calculated based on color, which is a measure of the dissimilarity between neighboring elements $v_{i}$ and $v_{j}$.

A minimum spanning tree is obtained using Kruskal's algorithm [36]. The connected components that are obtained represent image regions. It is supposed that the graph has $m$ edges and $n$ vertices. This algorithm is described also in [39] where it has four major steps that are presented below:

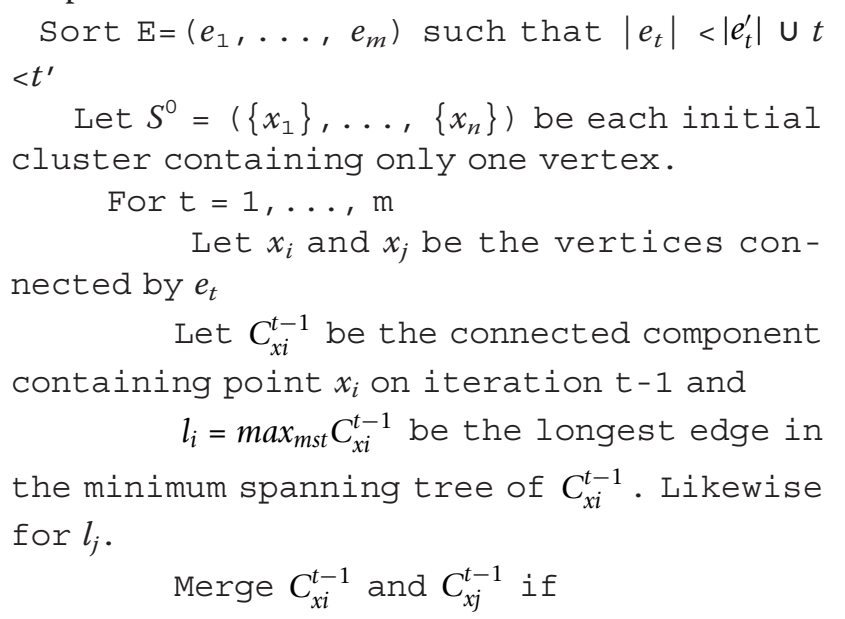

$$
\left|e_{t}\right|<\min \left\{l_{i}+\frac{k}{C_{x i}^{t-1}}, l_{j}+\frac{k}{C_{x j}^{t-1}}\right\} \text { where } \mathrm{k} \text { is a }
$$

constant

$$
S=S^{m}
$$

The existence of a boundary between two components in segmentation is based on a predicate $D$. This predicate is measuring the dissimilarity between elements along the boundary of the two components relative to a measure of the dissimilarity among neighboring elements within each of the two components. The internal difference of a component $C \subseteq V$ was defined as the largest weight in the minimum spanning tree of a component $\operatorname{MST}(C, E)$ :

$$
\operatorname{Int}(C)=\max _{e \in M S T(C E)} w(e)
$$

A threshold function is used to control the degree to which the difference between components must be larger than minimum internal difference. The pairwise comparison predicate is defined as:

$$
D\left(C_{1}, C_{2}\right)=\left\{\begin{array}{l}
\text { true, ifDif }\left(C_{1}, C_{2}\right)>\operatorname{MInt}\left(C_{1}, C 2\right) \\
\text { false, otherwise }
\end{array}\right.
$$

where the minimum internal difference Mint is defined as:

$$
\operatorname{MInt}\left(C_{1}, C_{2}\right)=\min \left(\operatorname{Int}\left(C_{1}\right)+\tau\left(C_{1}\right), \operatorname{Int}\left(C_{2}\right)+\tau\left(C_{2}\right)\right)
$$

The threshold function was defined based on the size of the component: $\tau(C)=k /|C|$. The $k$ value is set taking into account the size of the image. For images having the size $128 \times 128 k$ is set to 150 and for images with size $320 \times 240 k$ is set to 300 . The algorithm for creating the minimum spanning tree can be implemented to run in $O(m \log m)$ where $m$ is the number of edges in the graph. The input of the algorithm is represented by a graph $G=(V, E)$ with $n$ vertices and $m$ edges. The obtained output is a segmentation of $V$ in the components $S=\left(C_{1}, \ldots, C_{r}\right)$. The algorithm has five major steps:

Sort $\mathrm{E}$ into $\pi=\left(o_{1}, o_{t \pi}\right)$ by non-decreasing edge weight

Start with a segmentation $S^{D}$, where each vertex $v_{i}$ is in own component

Repeat step 4 for $\$ q=1, . . ., \mathrm{m} \$$

Construct $S^{q}$ using $S^{q}{ }_{-1}$ and the internal difference. If $v_{i}$ and $v_{j}$ are in disjoint components of $S_{-1}^{q}$ and the weight of the edge between $v_{i}$ and $v_{j}$ is small compared to the internal difference then merge the two components otherwise do nothing.

Return $S=S^{t \pi}$

Unlike the classical methods this technique adaptively adjusts the segmentation criterion based on the degree of variability in neighboring regions of the image. 


\section{Segmentation error measures}

A potential user of an algorithm's output needs to know what types of incorrect/invalid results to expect, as some types of results might be acceptable while others are not. This called for the use of metrics that are necessary for potential consumers to make intelligent decisions.

This section presents the characteristics of the error metrics defined in [4]. The authors proposed two metrics that can be used to evaluate the consistency of a pair of segmentations, where segmentation is simply a division of the pixels of an image into sets. Thus a segmentation error measure takes two segmentations S1 and $\mathrm{S} 2$ as input and produces a real valued output in the range $[0 \ldots 1]$ where zero signifies no error.

The process defines a measure of error at each pixel that is tolerant to refinement as the basis of both measures. A given pixel pi is defined in relation to the segments in S1 and S2 that contain that pixel. As the segments are sets of pixels and one segment is a proper subset of the other, then the pixel lies in an area of refinement and the local error should be zero. If there is no subset relationship, then the two regions overlap in an inconsistent manner. In this case, the local error should be non-zero. Let $\backslash$ denote set difference, and $|x|$ the cardinality of set $x$. If $\mathrm{R}(\mathrm{S}, \mathrm{pi})$ is the set of pixels corresponding to the region in segmentation $\mathrm{S}$ that contains pixel pi, the local refinement error is defined as in [4]:

$$
E(S 1, S 2, p i)=\frac{|R(S 1, p i) / R(S 2, p i)|}{|R(S 1, p i)|}
$$

Note that this local error measure is not symmetric. It encodes a measure of refinement in one direction only: $E(S 1, S 2, p i)$ is zero precisely when $S 1$ is a refinement of $\mathrm{S} 2$ at pixel pi, but not vice versa. Given this local refinement error in each direction at each pixel, there are two natural ways to combine the values into an error measure for the entire image. GCE forces all local refinements to be in the same direction. Let $n$ be the number of pixels:

$$
\operatorname{GCE}(S 1, S 2)=\frac{1}{n} \min \left\{\sum_{i} E(S 1, S 2, p i), \sum_{i} E(S 2, S 1, p i)\right\}
$$

LCE allows refinement in different directions in different parts of the image.

$$
\operatorname{LCE}(S 1, S 2)=\frac{1}{n} \sum_{i} \min \{E(S 1, S 2, p i), E(S 2, S 1, p i)\}
$$

As $\mathrm{LCE} \leq \mathrm{GCE}$ for any two segmentations, it is clear that GCE is a tougher measure than LCE. Martin et al. showed that, as expected, when pairs of human segmentations of the same image are compared, both the GCE and the LCE are low; conversely, when random pairs of human segmentations are compared, the resulting GCE and LCE are high.

\section{Experiments and results}

This section presents the experimental results for the evaluation of the three segmentation algorithms and error measures values.

The experiments were made on a database with 500 medical images from digestive area captured by an endoscope. The images were taken from patients having diagnoses such as polyps, ulcer, esophagites, colitis, and ulcerous tumors.

For each image, the following steps are performed by the application that we have created to calculate the GCE and LCE values:

Obtain the image regions using the color set backprojection segmentation

Obtain the image regions using the LV

Obtain the image regions using the algorithm based on the HS

Obtain the manually segmented regions

Store these regions in the database

Calculate GCE and LCE

Store these values in the database for later statistics

In Figure 2 the images for which we present some experimental results are presented. Figures 3 and 4 present the regions resulted from manual segmentation and from the application of the three algorithms presented above for the images displayed in Figure 2.

In Table 1 the number of regions resulted from the application of the segmentation can be seen.

In Table 2 the GCE values calculated for each algorithm are presented.

In Table 3 the LCE values calculated for each algorithm are presented.

If two different segmentations arise from different perceptual organizations of the scene, then it is fair to declare the segmentations inconsistent. If, however, segmentation is simply a refinement of the other, then the error should be small, or even zero. The error measures presented in the above tables are calculated in relation with the manual segmentation which is considered true segmentation. From Tables 2 and 3 it can be observed that the values for GCE and LCE are lower in the case of hexagonal segmentation. The error measures, for almost all tested images, have smaller values in the case of the original segmentation method which use a HS defined on the set of pixels.

Figure 5 presents the repartition of the 500 images from the database on GCE values. The focus point here is the number of images on which the GCE value is under 0.5 . 

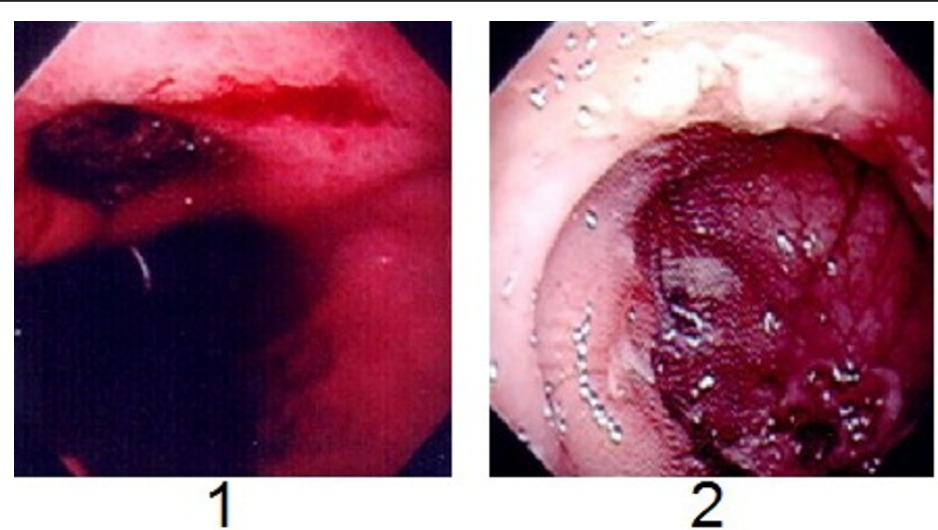

Figure 2 Images used in experiments

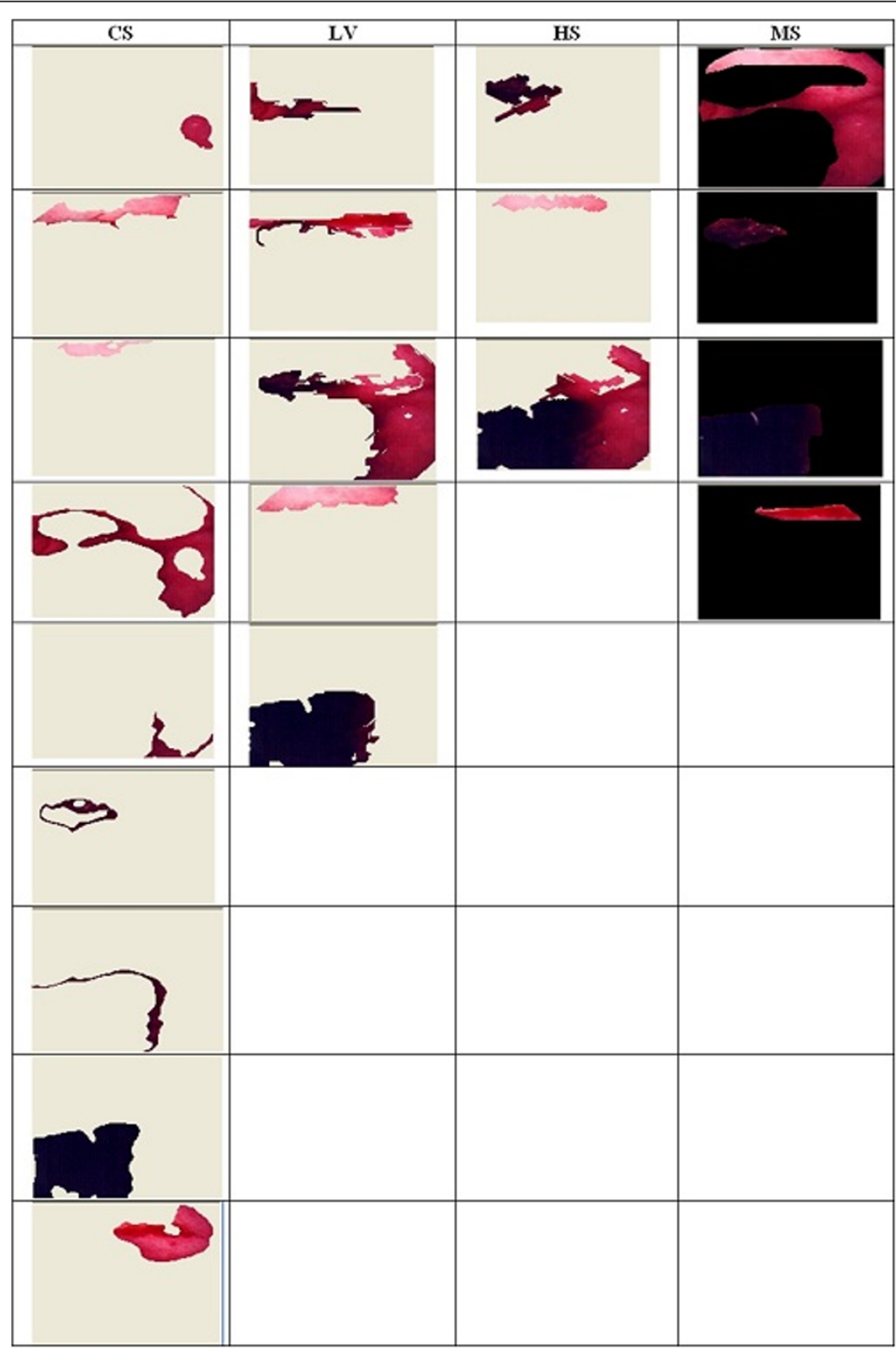

Figure 3 The resulted regions for image no. 1 


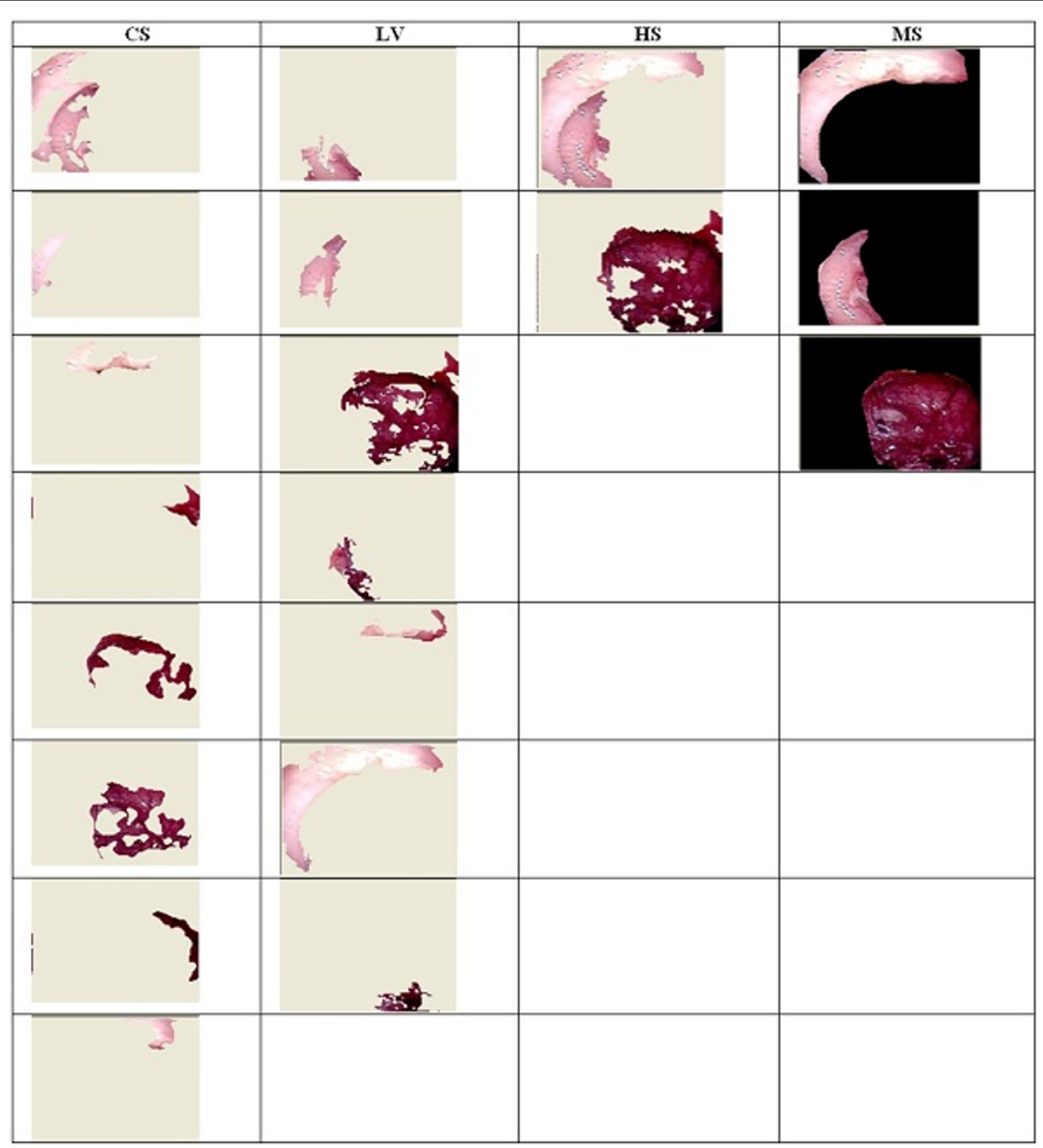

Figure 4 The resulted regions for image no. 2 .

In conclusion, for HS algorithm, a number of 391 images (78\%) obtained GCE values under 0.5. Similarly, for CS algorithm only 286 images (57\%) obtained GCE values under 0.5 . The segmentation based on LV method is close to our original algorithm: 382 images (76\%) had GCE values under 0.5.

Because the error measures for segmentation using a HS defined on the set of pixels are lower than for color set back-projection and local variation segmentation, we can infer that the segmentation method based on HS is more efficient.

Table 1 The number of regions detected for each algorithm

\begin{tabular}{lllll}
\hline Image number & CS & LV & HS & MS \\
\hline 1 & 9 & 5 & 3 & 4 \\
2 & 8 & 7 & 2 & 3 \\
\hline
\end{tabular}

Experimental results show that the original segmentation method based on a HS is a good refinement of the manual segmentation.

\section{Conclusion}

The aim of this article is to evaluate three algorithms able to detect the regions in endoscopic images: a

Table 2 GCE values calculated for each algorithm

\begin{tabular}{llll}
\hline Image number & GCE-CS & GCE-LV & GCE-HS \\
\hline 1 & 0.18 & 0.24 & 0.09 \\
2 & 0.36 & 0.28 & 0.10 \\
\hline
\end{tabular}

Table 3 LCE values calculated for each algorithm

\begin{tabular}{llll}
\hline Image number & LCE-CS & LCE-LV & LCE-HS \\
\hline 1 & 0.11 & 0.15 & 0.07 \\
2 & 0.18 & 0.17 & 0.12 \\
\hline
\end{tabular}




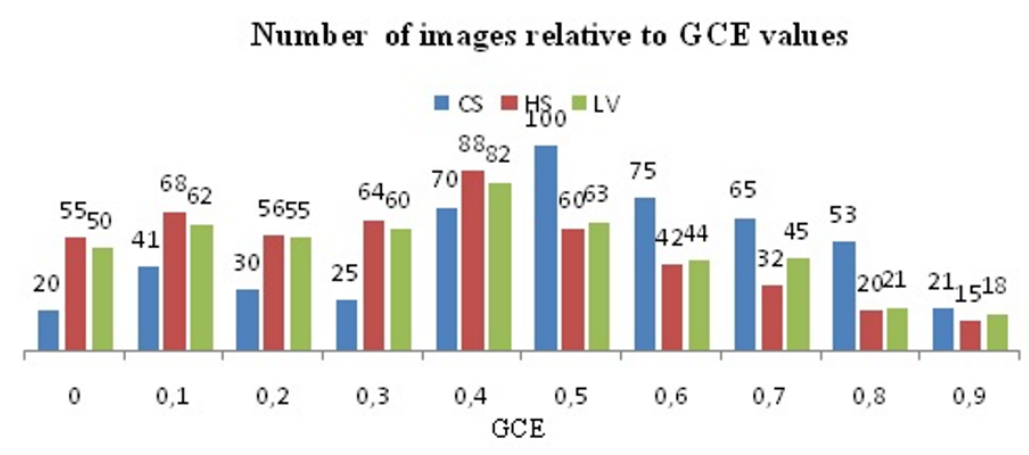

Figure 5 Number of images relative to GCE values.

clustering method (the color set back-projection algorithm), as well as other two methods of segmentation based on graphs: the LV and our original segmentation algorithm.

Our method is based on an HS defined on the set of image pixels. The advantage of using a virtual hexagonal network superposed over the initial image pixels is that it reduces the execution time and the memory space used, without loosing the initial resolution of the image. In comparison to other segmentation methods, our algorithm is able to adapt and does not require neither parameters for establishing the optimal values, nor sets of training images to set parameters.

Furthermore, the article presents a method that enables the assessment of the segmentation accuracy.

The role of this review is to find out which segmentation algorithm gives the best results for medical images from digestive area captured by an endoscope.

First, the correctness of segments resulted after the application of the three algorithms described above is compared. Concerning the endoscopic database all the algorithms have the ability to produce segmentations that comply with the manual segmentation made by a medical expert. Then for evaluating the accuracy of the segmentation error measures are used. The proposed error measures quantify the consistency between segmentations of differing granularities. Because human segmentation is considered true segmentation the error measures are calculated in relation with manual segmentation. The GCE and LCE demonstrate that the image segmentation based on an HS produces a better segmentation than the back-projection method and the LV.

The future research will focus on developing our segmentation algorithm so as to include the texture feature along with the color feature and reducing the algorithm complexity at $O(n \log n)$, where $n$ represents the number of image pixels.

\section{Acknowledgements}

The support of The National University Research Council under Grant CNCSIS IDEI 535 is gratefully acknowledged.

\section{Competing interests}

The authors declare that they have no competing interests.

Received: 15 May 2011 Accepted: 9 December 2011

Published: 9 December 2011

\section{References}

1. DL Pham, C Xu, JL Prince, Current methods in medical image segmentation. Annu Rev Biomed Eng. 2, 315-337 (2000). doi:10.1146/ annurev.bioeng.2.1.315

2. L Stanescu, D Burdescu, M Brezovan, in Chapter Book: Multimedia Medical Databases, Biomedical Data and Applications, Series: Studies in Computational Intelligence, vol. 224, ed. by Sidhu AS, Dillon T, Bellgard M (Springer, 2009)

3. SK Warfield, KH Zou, WM Wells, Simultaneous truth and performance level estimation (STAPLE): an algorithm for the validation of image segmentation Med Imag IEEE Trans. 23(7), 903-921 (2004). doi:10.1109/TMI.2004.828354

4. D Martin, C Fowlkes, D Tal, J Malik, A database of human segmented natural images and its application to evaluating segmentation algorithms and measuring ecological statistics, in Proceedings of the Eighth International Conference On Computer Vision (ICCV-01). 2, 416-425 (2001)

5. DD Burdescu, $\mathrm{L}$ Stanescu, A new algorithm for content-based region query in databases with medical images, in Conference of International Council on Medical and Care Compunetics, Symposium On Virtual Hospitals, Symposium on E-Health, Proceedings in Studies In Health Technology and Informatics. 114 132-133 (2005). Medical and Care Compunetics 2

6. DD Burdescu, $\mathrm{L}$ Stanescu, A new algorithm for content-based region query in multimedia database, in 16th International Conference on Database and Expert Systems Applications, Proceedings in Lectures Notes in Computer Science. 3588, 124-133 (2005)

7. DD Burdescu, L Stanescu, Color image segmentation applied to medical domain. Intell Data Eng Autom Learn. 4881, 457-466 (2007). doi:10.1007/ 978-3-540-77226-2_47

8. L Stanescu, DD Burdescu, Medical image segmentation-a comparison of two algorithms, in International Workshop on Medical Measurements and Applications (2010)

9. JR Smith, Integrated spatial and feature image systems: retrieval, compression and analysis, PhD thesis, Graduate School of Arts and Sciences Columbia University, (1997)

10. H Muller, N Michoux, D Bandon, A Geissbuhler, A review of content-based image retrieval systems in medical applications-clinical benefits and future directions. Int J Med Inf. 73(1), 1-23 (2004). doi:10.1016/j. ijmedinf.2003.11.024

11. C Jiang, X Zhang, M Christoph, Hybrid framework for medical image segmentation. Lecture Notes in Computer Science. 3691, 264-271 (2005). doi:10.1007/11556121_33 
12. S Belongie, C Carson, H Greenspan, J Malik, Color and texture-based image segmentation using the expectation-maximization algorithm and its application to content-based image retrieval. in ICCV 675-682 (1998)

13. C Carson, S Belongie, H Greenspan, J Malik, Blobworld: image segmentation using expectation-maximization and its application to image querying. IEEE Trans Pattern Anal Mach Intell. 24(8), 1026-1038 (2002). doi:10.1109/ TPAMI.2002.1023800

14. L Ballerini, Medical image segmentation using Genetic snakes, Applications and Science of Neural Networks, Fuzzy Systems, and Evolutionary Computation II, (Denver Co, 1999)

15. S Ghebreab, AWM Smeulders, Medical images segmentation by strings, in Proceedings of the VISIM Workshop: Information Retrieval and Exploration in Large Collections of Medical Images (2001)

16. S Ghebreab, AWM Smeulders, An approximately complete string representation of local object boundary features for concept-based image retrieval, in IEEE International Symposium on Biomedical Imaging (2004)

17. S Gordon, G Zimmerman, H Greenspan, Image segmentation of uterine cervix images for indexing in PACS, in Proceedings of the 17th IEEE Symposium on Computer-Based Medical Systems, CBMS (2004)

18. H Lamecker, T Lange, M Seeba, S Eulenstein, M Westerhoff, HC Hege, Automatic segmentation of the liver for preoperative planning of resections, in Proceedings of Medicine Meets Virtual Reality, Studies in Health Technologies and Informatics. 94, 171-174 (2003)

19. H Muller, S Marquis, G Cohen, PA Poletti, CL Lovis, A Geissbuhler, Automatic abnormal region detection in lung $C T$ images for visual retrieval. Swiss Med Inf. 57, 2-6 (2005)

20. PF Felzenszwalb, WD Huttenlocher, Efficient graph-based image segmentation. Int J Comput Vis. 59(2), 167-181 (2004)

21. L Guigues, LM Herve, LP Cocquerez, The hierarchy of the cocoons of a graph and its application to image segmentation. Pattern Recogn Lett. 24(8), 1059-1066 (2003). doi:10.1016/S0167-8655(02)00252-0

22. J Shi, J Malik, Normalized cuts and image segmentation. in Proceedings of the IEEE Conference on Computer Vision and Pattern Recognition 731-737 (1997)

23. Z Wu, R Leahy, An optimal graph theoretic approach to data clustering: theory and its application to image segmentation. IEEE Trans Pattern Anal Mach Intell. 15(11), 1101-1113 (1993). doi:10.1109/34.244673

24. C Zahn, Graph-theoretical methods for detecting and describing gestal clusters. IEEE Trans Comput. 20(1), 68-86 (1971)

25. R Urquhar, Graph theoretical clustering based on limited neighborhood sets. Pattern Recogn. 15(3), 173-187 (1982). doi:10.1016/0031-3203(82) 90069-3

26. Y Gdalyahu, D Weinshall, M Werman, Self-organization in vision: stochastic clustering for image segmentation, perceptual grouping, and image database organization. IEEE Trans Pattern Anal Mach Intell. 23(10), 1053-1074 (2001). doi:10.1109/34.954598

27. I Jermyn, H Ishikawa, Globally optimal regions and boundaries as minimum ratio weight cycles. IEEE Trans Pattern Anal Mach Intell. 23(8), 1075-1088 (2001)

28. M Cooper, The tractibility of segmentation and scene analysis. Int J Comput Vis. 30(1), 27-42 (1998). doi:10.1023/A:1008013412628

29. T Pavlidis, Structural Pattern Recognition (Springer, 1977)

30. D Comaniciu, P Meer, Robust analysis of feature spaces: color image segmentation. in Proceedings of the IEEE Conference on Computer Vision and Pattern Recognition 750-755 (2003)

31. A Jain, R Dubes, Algorithms for Clustering Data (Prentice Hall, 1988)

32. L Stanescu, Visual Information. Procecessing, Retrieval and Applications (Sitech, Craiova, 2008)

33. A Gijsenij, T Gevers, M Lucassen, A perceptual comparison of distance measures for color constancy algorithms, in Proceedings of the European Conference on Computer Vision. 5302, 208-2011 (2008)

34. X Zhang, X Wu, Image coding on quincunx lattice with adaptive lifting and interpolation. in Data Compression Conference 193-202 (2007)

35. DD Burdescu, M Brezovan, E Ganea, L Stanescu, A new method for segmentation of images represented in a HSV color space. in ACIVS 606-617 (2009)

36. MT Goodrich, R Tamassia, Data Structures and Algorithms in Java, 4th edn. (John Wiley and Sons, Inc, 2006)

37. M Brezovan, DD Burdescu, E Ganea, L Stanescu, Boundary-based performance evaluation of a salient visual object detection algorithm, in Proceedings of the WorldComp IPCV (2010)
38. JR Smith, SF Chang, Tools and techniques for color image retrieval, Proc SPIE Storage retrieval for still image and video databases, IV. 2670, 426-437 (1996)

39. C Pantofaru, M Hebert, A comparison of image segmentation algorithms, Technical Report CMU-RI-TR-05-40, Robotics Institute, Carnegie Mellon University, Pittsburgh, PA, (2005)

doi:10.1186/1687-6180-2011-128

Cite this article as: Stanescu et al:: A comparative study of some methods for color medical images segmentation. EURASIP Journal on Advances in Signal Processing 2011 2011:128.

\section{Submit your manuscript to a SpringerOpen ${ }^{\circ}$ journal and benefit from:}

- Convenient online submission

- Rigorous peer review

- Immediate publication on acceptance

- Open access: articles freely available online

- High visibility within the field

- Retaining the copyright to your article

Submit your next manuscript at $>$ springeropen.com 